\title{
Optimal user pairing for multiuser MIMO
}

\author{
Emanuele Viterbo \\ D.E.I.S. \\ Università della Calabria \\ Arcavacata di Rende, Italy \\ Email: viterbo@deis.unical.it
}

\author{
Ari Hottinen \\ Nokia Research Center \\ Helsinki, Finland \\ Email: ari.hottinen@nokia.com
}

\begin{abstract}
In this paper we show how the capacity of the uplink of a multiuser system can be increased by a scheduling strategy, which pairs the transmission of users in different time/frequency/code slots according to the channel quality. The optimal scheduling strategy is equivalent to a combinatorial optimization problem. We show how this problem can be solved efficiently by using the Hungarian method. We then show that, by using the proposed scheduling scheme, the performance of Minimum Mean Square Error detection approaches the one of Maximum Likelihood detection, as the number of users increases.
\end{abstract}

Index terms - Capacity, Scheduling, multiuser uplink, MIMO, assignment problem, Hungarian method.

\section{INTRODUCTION}

A multiuser multiple-input-multiple output (MU-MIMO) system consists of $K$ user with $n_{t}$ antennas each communicating to a base station with $n_{r}$ receive antennas. Since each user faces a different channel condition, in different time/frequency/code (TFC) slots it is possible to improve the overall system capacity by multiuser scheduling. Multiuser scheduling has been applied previously in allocating different MIMO users mutually orthogonal sum-rate-optimized subchannels, see e.g. [4]. Here, we consider a system where different users access the channel in the same slot, and thus cause interference to each other. This method is supported by IEEE 802.16e specification under the name Collaborative Spatial Multiplexing. However, optimal user scheduling is not addressed in this specification.

Multiuser scheduling, as considered here, attempts to increase the system capacity by smartly allocating the channel to different subgroups of users. A general introduction to this topic can be found in [1] and some related scheduling algorithms for uplink and downlink can be found in [2], [5]. The most popular multiuser scheduling schemes include opportunistic scheduling and best subset selection. All scheduling schemes are confronted with the fairness issue. Fairness requirement, however defined, typically enforces a tradeoff between network optimality and user optimality.

In this paper we will focus on a fair scheduling scheme based on user pairing in uplink and assume as objective function the total instantaneous mutual information between users and the base station when both ML and MMSE receivers are considered. We first show that the combinatorial optimization problem, which yields an optimal scheduling, can be solved efficiently by using the Hungarian method [10], [11],
[12]. This scheduling scheme enforces fairness by letting each user transmit with the same total energy within a predefined multiuser scheduling window. We then show that, by using the proposed scheduling scheme, the performance of Minimum Mean Square Error (MMSE) detection approaches the one of Maximum Likelihood (ML) detection, as the number of users increases.

\section{SySTEM MODEL}

In this section, we describe the multiuser system model and we state the scheduling problem based on user pairing as a combinatorial optimization problem.

Considering the uplink channel, we assume that the users are multiplexed in the code domain, i.e., all user's signals overlap both in time and in frequency within a channel use. For $K$ users we have

$$
\mathbf{y}=\sum_{k=1}^{K} \mathbf{H}^{(k)} \mathbf{x}^{(k)}+\mathbf{z}
$$

where $\mathbf{x}^{(k)} \in \mathbb{C}^{n_{t}}$ is the transmitted column vector from user $k, \mathbf{H}^{(k)} \in \mathbb{C}^{n_{r} \times n_{t}}$ the channel coefficient matrix, $\mathbf{z} \in \mathbb{C}^{n_{r}}$ the white Gaussian noise vector distributed as $\mathcal{N}_{c}\left(0, \mathbf{I}_{n_{r}}\right)$. Let $P$ be the total transmitted power by each user (i.e., $P=\mathbb{E}\left[\left\|\mathbf{x}^{(k)}\right\|^{2}\right]$ ), then we define $\mathrm{SNR}=P$.

We assume the transmitter does not know the channel (open loop) and the receiver has knowledge of each user channel matrix. Furthermore, we assume that a power control scheme is used to compensate the path-loss, so that the average received power from each user is balanced and equal to $P$.

Let us rewrite (1) in equivalent matrix form

$$
\mathbf{y}=\left[\mathbf{H}^{(1)}|\cdots| \mathbf{H}^{(K)}\right]\left[\begin{array}{c}
\mathbf{x}^{(1)} \\
\vdots \\
\mathbf{x}^{(K)}
\end{array}\right]+\mathbf{z}=\mathcal{H} \mathbf{X}+\mathbf{z}
$$

where we assume that the joint channel $n_{r} \times K n_{t}$ matrix $\mathcal{H}$ is constant during the channel use and $\mathbf{X}$ is the joint input vector of length $K n_{t}$.

Assuming the receiver performs ML detection the mutual information per user (conditioned by the channel realization) for channel (2) is given by

$$
I_{M L}(\mathbf{X} ; \mathbf{y} \mid \mathcal{H})=\frac{1}{K} \log _{2}\left(\operatorname{det}\left(\mathbf{I}_{n_{r}}+\frac{P}{K n_{t}} \mathcal{H} \mathcal{H}^{\dagger}\right)\right)
$$


Due to the high complexity of ML detection, the simpler MMSE receiver is generally adopted, and in this case we have

$$
I_{M M S E}(\mathbf{X} ; \mathbf{y} \mid \mathcal{H})=\frac{1}{K} \sum_{j=1}^{K n_{t}} \log _{2}\left(1+\mathbf{h}_{j}^{\dagger} \mathbf{A}_{j}^{-1} \mathbf{h}_{j}\right)
$$

where $\mathbf{h}_{j}$ are the column vectors of $\mathcal{H}$ and

$$
\mathbf{A}_{j}=\frac{K n_{t}}{P} \mathbf{I}_{n_{r}}+\sum_{i=1, i \neq j}^{K n_{t}} \mathbf{h}_{i}^{\dagger} \mathbf{h}_{i} .
$$

The above expressions represent a measure of the per-user throughput, given that the system is occupying a total bandwidth $B$.

The above scheme requires a $K$ user multiuser detection which can be still rather complex for large numbers of users and transmit antennas. For this reason it is common to consider joint TDMA/FDMA/CDMA/SDMA schemes to reduce the number of simultaneous users by allocating them in different TFC slots within a frame. Since the channel matrices for the users are different and determine how the users signals interfere at the receiver, scheduling the users that simultaneously transmit in the same TFC slot, can improve the total system throughput.

\section{A. Pairing users}

Let us first consider the case where $K$ is even and users are paired to transmit simultaneously in the same TFC slot. The total number of TFC slots (or channel orthogonal resources) is then $N=K / 2$ and we assume the total occupied bandwidth is still $B$. Fairness is provided by the fact that all users access the channel exactly once, within a frame of $N$ TFC slots. We let $\mathbf{H}^{(k)}$ denote the channel for user $k$ and assume it is constant over the entire frame.

In this case we have that the received signal in the $n$-th TFC slot is

$\mathbf{y}(n)=\sum_{\left(k_{1}, k_{2}\right)}^{(N)} \mathbf{H}^{\left(k_{1}\right)} \mathbf{x}^{\left(k_{1}\right)}+\mathbf{H}^{\left(k_{2}\right)} \mathbf{x}^{\left(k_{2}\right)}+\mathbf{z}(n) \quad n=1, \ldots, N$

where the sum runs over $N$ distinct pairs $\left(k_{1}, k_{2}\right)$ of users, with $k_{1} \neq k_{2}$. Note that the multiuser detection now handles only two overlapping users per TFC slot and thus even multiuser ML detection could become viable.

We denote by $\pi$ a particular pairing configuration, within the set of all configurations $\Pi$. The number of ways to choose $N$ disjoint pairs of items from $2 N$ items is ([7])

$$
|\Pi|=(2 N-1) ! !=(2 N-1)(2 N-3) \cdots 31 .
$$

For example, with $K=4$ users we have three configurations

$$
\Pi=\{\{(12),(34)\},\{(13)(24)\},\{(14)(23)\}\}
$$

Given (5), the per-user mutual information between $\mathbf{X}$ and $\mathbf{Y}=\left(\mathbf{y}(1)^{T}, \ldots, \mathbf{y}(N)^{T}\right)^{T}$, given a pairing configuration $\pi$, is

$$
\begin{aligned}
& I_{M L}(\mathbf{X} ; \mathbf{Y} \mid \mathcal{H}, \pi)= \\
& \quad \frac{1}{N} \sum_{\left(k_{1}, k_{2}\right) \in \pi}^{(N)} \log _{2}\left(\operatorname{det}\left(\mathbf{I}_{n_{r}}+\frac{P}{2 n_{t}} \mathbf{H}^{\left(k_{1}, k_{2}\right)} \mathbf{H}^{(k 1, k 2)^{\dagger}}\right)\right)
\end{aligned}
$$

where $\mathbf{H}^{\left(k_{1}, k_{2}\right)}=\left[\mathbf{H}^{\left(k_{1}\right)} \mid \mathbf{H}^{\left(k_{2}\right)}\right]$. Similarly

$$
\begin{aligned}
& I_{M M S E}(\mathbf{X} ; \mathbf{Y} \mid \mathcal{H}, \pi)= \\
& \frac{1}{N} \sum_{\left(k_{1}, k_{2}\right)}^{(N)} \sum_{j=1}^{2 n_{t}} \log _{2}\left(1+\mathbf{h}_{j}^{(k 1, k 2)^{\dagger}} \mathbf{A}_{j}^{(k 1, k 2)^{-1}} \mathbf{h}_{j}^{(k 1, k 2)}\right)
\end{aligned}
$$

where $\mathbf{h}_{j}^{(k 1, k 2)}$ are the $2 n_{t}$ columns of $\mathbf{H}^{\left(k_{1}, k_{2}\right)}$ and

$$
\mathbf{A}_{j}^{(k 1, k 2)}=\frac{2 n_{t}}{P} \mathbf{I}_{n_{r}}+\sum_{i=1, i \neq j}^{2 n_{t}} \mathbf{h}_{i}^{(k 1, k 2)^{\dagger}} \mathbf{h}_{i}^{(k 1, k 2)} .
$$

Both (6) and (7) can be written as additive objective functions to be maximized over the choice of $\pi \in \Pi$

$$
\max _{\pi \in \Pi} \sum_{\left(k_{1}, k_{2}\right) \in \pi}^{(N)} f_{k_{1}, k_{2}}(\pi)
$$

Selecting the pairing configuration that maximizes the above mutual information can become a formidable task even for a small number of users due to the exponential complexity of an exhaustive search. For example, for $K=2,4,6,8,10,16$ we have a number of configurations $|\Pi|=1,3,15,105,945,2027025$. We will show in Sec. II-D how this problem can be solved in polynomial time using a technique known as Hungarian method.

\section{B. Both single users and paired users}

We now consider the case where we allow some users to transmit alone and some others to be paired in the TFC slots. The total number of users is $K=2 N_{\text {pair }}+N_{\text {sing }}$, where $N_{\text {pair }}$ is the number of pairs of users that transmit simultaneously in a TFC slot and $N_{\text {sing }}$ is the number of users that transmit alone. In this case the total number of TFC slots used in one transmission frame would be $N=N_{\text {pair }}+N_{\text {sing }}$ and the total number of configurations $\Pi$ is much larger than before, namely:

$$
|\Pi|=\sum_{k=0}^{\lfloor K / 2\rfloor} \frac{K !}{(K-2 k) ! 2^{k} k !}
$$

This number corresponds to the number of partitions of a set of $K$ distinguishable elements into sets of size 1 and 2 or equivalently to the number of $K \times K$ symmetric permutation matrices [8]. For example, for $K=2,4,6,8,16$ we have $|\Pi|=2,10,76,764,46206736$ and with $K=4$ users we have the following 10 configurations

$$
\begin{aligned}
\Pi= & \{\{(12)(34)\},\{(13)(24)\},\{(14)(23)\}, \\
& \{(1)(2)(34)\},\{(1)(3)(24)\},\{(1)(4)(23)\}, \\
& \{(12)(3)(4)\},\{(13)(2)(4)\},\{(14)(2)(3)\}, \\
& \{(1)(2)(3)(4)\}\}
\end{aligned}
$$


In this case the optimization problem becomes

$$
\begin{array}{r}
\max _{\pi \in \Pi}\left\{\frac{1}{N_{\text {pair }}(\pi)} \sum_{\left(k_{1}, k_{2}\right) \in \pi}^{\left(N_{\text {pair }}(\pi)\right)} f_{k_{1}, k_{2}}^{(\text {pair })}(\pi)+\right. \\
\left.\frac{1}{N_{\text {sing }}(\pi)} \sum_{\left(k_{3}\right) \in \pi}^{\left(N_{\text {sing }}(\pi)\right)} f_{k_{3}}^{(\text {sing })}(\pi)\right\}
\end{array}
$$

We can think of the single users $\left(k_{3}\right)$ as paired with themselves, i.e., $\left(k_{3}, k_{3}\right)$. Unfortunately, this problem cannot be solved by the Hungarian method, since the objective function is not a sum of terms only depending on one pair due to the factors $\frac{1}{N_{\text {pair }}(\pi)}$ and $\frac{1}{N_{\text {sing }}(\pi)}$ (see Section II-D for details). Due to the exponential complexity required to solve 9 we are motivated to consider the new scheduling scheme of the following section.

\section{New scheduling scheme}

In order to have the same total bandwidth for all configurations with different $N_{\text {pair }}$ and $N_{\text {sing }}$, we assume that the $N_{\text {pair }}$ paired users access two TFC slots, essentially doubling their rate. As a compensation, the $N_{\text {sing }}$ unpaired users, that only use one TFC slot, are allowed to double their transmit power. This will produce comparable out-ofcell interfering power during all TFC slots. Now the total number of TFC slots used in one transmission frame would be $N=2 N_{\text {pair }}+N_{\text {sing }}=K$. By transmitting with double power, unpaired users can employ a higher order modulation in order to double their spectral efficiency and compensate for their use of only one TFC slot.

Let us now show how a pairing configuration $\pi=$ $\left\{\pi_{\text {pair }}, \pi_{\text {sing }}\right\}$ can be mapped to a permutation $\sigma$ of $K$ elements of the form

$$
\sigma: \quad\left(\begin{array}{cccc}
1 & 2 & \cdots & K \\
\sigma(1) & \sigma(2) & \cdots & \sigma(K)
\end{array}\right) .
$$

Let the pairs $\left(k_{1}, k_{2}\right) \in \pi_{\text {pair }}$ correspond to the two columns of (10) $\left(k_{1}, k_{2}=\sigma\left(k_{1}\right)\right)^{T}$ and $\left(k_{2}, k_{1}=\sigma\left(k_{2}\right)\right)^{T}$, while the unpaired users $\left(k_{3}\right) \in \pi_{\text {sing }}$ correspond to the fixed elements of the permutation, i.e., columns of (10) of the type $\left(k_{3}, k_{3}\right)^{T}$. For example

$$
\pi=\{(1,5)(2,4)(3)\} \quad \Rightarrow \quad \sigma: \quad\left(\begin{array}{ccccc}
1 & 2 & 3 & 4 & 5 \\
5 & 4 & 3 & 2 & 1
\end{array}\right)
$$

Clearly, under the assumptions of the Sections II-A and II-B this will limit the permutations $\sigma$ to have at most cycles of length 2 of the type $\left(k_{1}, k_{2}\right)$, [9].

In this new scenario we can further expand the possible pairing configurations to include any user permutation $\sigma$, i.e., we will consider $K$ pairs of users $(k, \sigma(k))$. For example we can have

$$
\begin{aligned}
& \pi=\{(1,5)(2,4)(3,3)(4,5)(5,2)\} \\
& \Rightarrow \quad \sigma:\left(\begin{array}{ccccc}
1 & 2 & 3 & 4 & 5 \\
5 & 4 & 3 & 1 & 2
\end{array}\right)
\end{aligned}
$$

which is a permutation with a cycle $(1,5,2,4)$ of length 4 .
The optimization problem can now be written as

$$
\max _{\sigma \in S_{K}} \sum_{(k, \sigma(k))}^{(K)} f_{(k, \sigma(k))}
$$

where $S_{K}$ denotes the group of all permutations (symmetric group).

\section{Solving the combinatorial optimization problem}

Here, we show how the above combinatorial optimization problems (8) and (11) can be solved in polynomial time $O\left(n^{3}\right)$ using a technique known as the Hungarian method commonly used to solve the so called assignment problem [10], [11], [12].

Assignment problem: Given a weighted complete bipartite graph $G=(X \cup Y ; X \times Y)$, where edge $x y$ has weight $w(x y)$, find a matching $M$ from $X$ to $Y$ with maximum weight.

In a common application, $X$ could be a set of workers, $Y$ could be a set of jobs, and $w(x y)$ could be the profit made by assigning worker $x$ to job $y$. By adding virtual jobs or workers with 0 profitability, we may assume that $X$ and $Y$ have the same size, $n$, and can be written as $X=\left\{x_{1} ; x_{2} ; \ldots, x_{n}\right\}$ and $Y=\left\{y_{1}, y_{2}, \ldots, y_{n}\right\}$.

Mathematically, the problem can be stated as follows: given an $n \times n$ matrix $W=\left[w_{k, \ell}\right]=\left[w\left(x_{k} y_{\ell}\right)\right]$, find a permutation $\sigma \in S_{n}$ of $n$ elements for which

$$
\sum_{k=1}^{n} w\left(x_{k} y_{\sigma(k)}\right)
$$

is a maximum. This form coincides with (11) when $w\left(x_{k} y_{\sigma(k)}\right)=f_{(k, \sigma(k))}$.

In order to solve the problem (8) in the case of even $K$, where no users are allowed to be unpaired it is enough to initialize the matrix $W$ with zero entries on the diagonal and symmetric entries $w_{k_{1}, k_{2}}=w_{k_{2}, k_{1}}=f_{\left(k_{1}, k_{2}\right)}$. The final solution is found by taking only the pairs $(k, \sigma(k))$, for $k=1, \ldots, K / 2$.

\section{PERFORMANCE}

In this section we demonstrate the gains provided by the proposed scheduling schemes in Sec. II-A and Sec. II-C. We quantify the average mutual information per user in a case where each of the $K$ users has an i.i.d. Rayleigh fading channel to the destination node. We assume $n_{t}=n_{r}=2$ and therefore at most two users may transmit concurrently.

The optimal user subsets for each slot are determined by solving the associated matching problem (via Hungarian algorithm) to maximize the sum capacity for $K$ users, where $K \in\{2,4,8,12,16\}$ in the considered examples. These optimal subsets are shown in figures with legend 'Optimal'. For comparison, we also depict the performance with random user pairing - these results are associated with legend 'Random'. With random pairing, we do not allow any single users. The case where only single users are allowed to access the channel sequentially is shown in figures with legend 'SU'. This corresponds to the case where the $k=\sigma(k), \forall k$. 


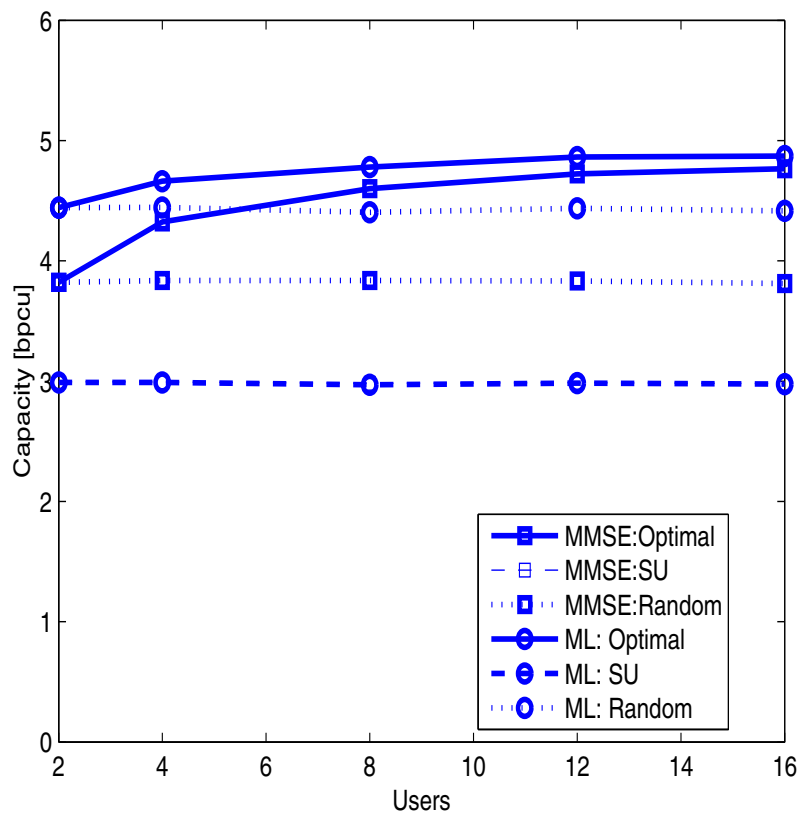

Fig. 1. Paired users only at SNR $=16 d B$. Note that ML and MMSE single user curves (two bottom curves) fully overlap.

In what follows, performance is evaluated for two receivers, for minimum mean square error receiver (legend 'MMSE') and for maximum likelihood receiver (legend 'ML'). For both receivers, we consider the scheduling schemes in Sec. II-A and Sec. II-C, and the reference cases stated above. We set $\mathrm{SNR}=16 d B$ when optimizing user pairing (no single users) and then $K / 2$ slots are scheduled. For comparison, we let $\mathrm{SNR}=13 d B$, when optimizing jointly over single users and pairs, since in this $K$ slots are scheduled. The difference in SNR ensures that for both schemes the total transmit energy over available slots is the same.

Figure 1 shows capacity expressed in bits per channel use (bpcu) as a function of the number of users $K$, when all the users are paired as for (8). The single user (SU) case, plotted for reference, coincides for MMSE and ML detection. Similar results are shown in Fig. 2 for the case (11).

The following observations are in order in both cases.

- There is a substantial capacity gain over the single user case, thanks to the spatial multiplexing and optimal pairing of the users.

- The gain of optimal scheduling increases for increasing number of users (for $K=2$ there is obviously no difference).

- The gain of optimal scheduling is larger for MMSE receiver since ML can handle better ill-conditioned situations.

- For large $K$ the MMSE seems to approach the ML capacity, provided that optimal users subsets are used.

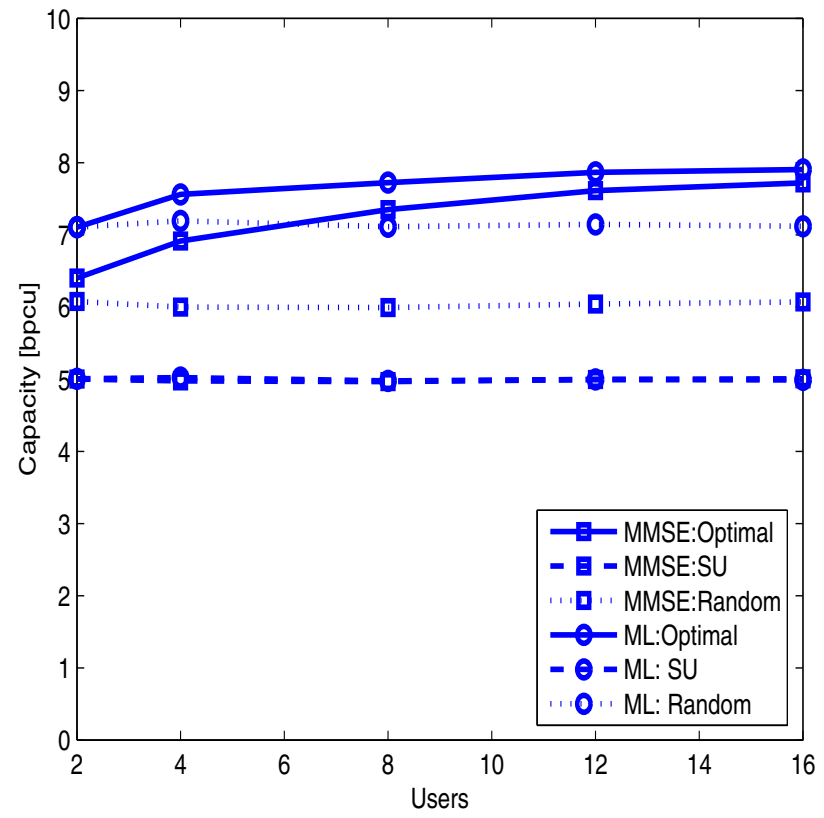

Fig. 2. Both pairs and single users at SNR $=13 d B$. Note that ML and MMSE single user curves (two bottom curves) fully overlap.

\section{CONCLUSIONS}

In this paper we have proposed a new computationally efficient and fair channel-aware multiuser scheduling scheme for uplink. In the considered scheduling scheme user subsets are optimized jointly, with polynomial complexity, over multiple transmission slots. In each slot at most two users are transmitting simultaneously. The proposed approach improves the performance of the multiuser system by efficiently exploiting channel and interference diversity and joint optimization over a given scheduling interval. It is shown via simulations that with the proposed scheduling method the MMSE receiver approaches, for large number of users, the performance of the ML receiver which has a higher complexity.

\section{REFERENCES}

[1] W. Ajib and D. Hoccoun, "An overview of scheduling algorithms in MIMO-based fourth-generation wireless systems," IEEE Network, Sept./Oct. 2005.

[2] M. Fuchs, , G. Del Galdo, and M. Haardt, "Low-complexity space-timefrequency scheduling for MIMO systems with SDMA," IEEE Tr. Vehic. Tech., Vol. 56, No. 5, pp. 2775-2784, Sept. 2005.

[3] R.W. Heath Jr., M. Airy, and A.J. Paulraj, "Multiuser diversity for MIMO wireless systems with linear receivers" Conference Record of the Thirty-Fifth Asilomar Conference on Signals, Systems and Computers, 4-7 Nov. 2001, Vol. 2, pp. 1194-1199.

[4] A. Hottinen, T. Heikkinen, "Subcarrier allocation in a multiuser MIMO channel using linear programming,"Proc. EUSIPCO 2006, Florence, Italy, Sept. 2006.

[5] A. Hottinen and E. Viterbo, "Optimal user pairing in downlink MUMIMO with transmit precoding," Proc. RAWNET, Berlin, Germany, March 2008.

[6] B. Bandemer, S. Visuri, "Capacity-Based Uplink Scheduling Using Long-Term Channel Knowledge," ICC '07. IEEE International Conference on Communications, 24-28 June 2007, pp. 785-790. 
[7] http://www.research.att.com/ njas/sequences/A001147

[8] http://www.research.att.com/ njas/sequences/A000085

[9] J.D. Dixon and B. Mortimer: Permutation Groups, Number 163 in Graduate Texts in Mathematics. Springer-Verlag, 1996.

[10] H.W. Kuhn, "The Hungarian Method for the assignment problem," Naval Research Logistic Quarterly, 2:83-97, 1955.

[11] H.W. Kuhn, "Variants of the Hungarian method for assignment problems," Naval Research Logistic Quarterly, 3: 253-258, 1956.

[12] J. Munkres, "Algorithms for the Assignment and Transportation Problems," Journal of the Society of Industrial and Applied Mathematics, 5(1):32-38, 1957 March.

[13] IEEE Std 802.16e, IEEE Standard for Local and metropolitan area networks, Part 16: Air Interface for Fixed and Mobile Broadband Wireless Access Systems, February 282006 